21st Particles and Nuclei International Conference (PANIC 2017)

International Journal of Modern Physics: Conference Series

Vol. 46 (2018) 1860041 (6 pages)

(C) The Author(s)

DOI: $10.1142 / \mathrm{S} 2010194518600418$

\title{
Recent Results from Borexino and Prospects for the SOX Experiment
}

\author{
Laura Collica \\ INFN Milano \\ Via Celoria 16, 20133 Milano, Italy \\ laura.collica@mi.infn.it
}

on behalf of the Borexino and SOX Collaboration:

M Agostini, K Altenmüller, S Appel, V Atroshchenko, Z Bagdasarian, D Basilico, G Bellini,

J Benziger, D Bick, G Bonfini, D Bravo, B Caccianiga, F Calaprice, A Caminata, S Caprioli,

M Carlini, P Cavalcante, A Chepurnov, K Choi, O Cloué, L Collica, M Cribier, D D'Angelo, S Davini,

A Derbin, X F Ding, A Di Ludovico, L Di Noto, I Drachnev, M. Durero, S Farinon, V Fischer,

K Fomenko, A Formozov, D Franco, F Gabriele, J Gaffiot, C Galbiati, M Gschwender, C Ghiano, M Giammarchi, A Goretti, M Gromov, D Guffanti, C Hagner, T Houdy, E Hungerford, Aldo Ianni, Andrea Ianni, N Jonquères, A Jany, D Jeschke, V Kobychev, D Korablev, G Korga, V Kornoukhov,

D Kryn, T Lachenmaier, T Lasserre, M Laubenstein, E Litvinovich, F Lombardi, P Lombardi,

L Ludhova, G Lukyanchenko, L Lukyanchenko, I Machulin, G Manuzio, S Marcocci, J Maricic,

G Mention, J Martyn, E Meroni, M Meyer, L Miramonti, M Misiaszek, V Muratova, R Musenich,

B Neumair, L Oberauer, B Opitz, V Orekhov, F Ortica, M Pallavicini, L Papp, Ö Penek, N Pilipenko,

A Pocar, A Porcelli, G Ranucci, A Razeto, A Re, M Redchuk, A Romani, R Roncin, N Rossi,

S Rottenanger, S Schönert, L Scola, D Semenov, M Skorokhvatov, O Smirnov, A Sotnikov,

L F F Stokes, Y Suvorov, R Tartaglia, G Testera, J Thurn, M Toropova, E Unzhakov, C Veyssiére,

A Vishneva, M Vivier, R B Vogelaar, F von Feilitzsch, H Wang, S Weinz, M Wojcik, M Wurm, Z Yokley, O Zaimidoroga, S Zavatarelli, K Zuber and G Zuzel

Published 3 May 2018

The Borexino experiment, located at Gran Sasso Laboratory in Italy, is operational since 2007. The unprecedented ultra-low background of the inner scintillating core has allowed to measure the fluxes of different components of the solar neutrino spectrum for the first time, as well as neutrino physical properties. In this work we present the recent results obtained with Borexino Phase-II data. Furthermore, we discuss the prospects for the SOX project, which will start at the beginning of 2018 and it will be able to test the long-standing issue of the existence of a sterile neutrino.

Keywords: Solar neutrino; sterile neutrino; Borexino.

\section{Introduction}

Borexino ${ }^{1}$ is devoted to perform high-precision neutrino observations, and is optimised for measurements in the low energy (subMeV) region of the solar neutrino

This is an Open Access article published by World Scientific Publishing Company. It is distributed under the terms of the Creative Commons Attribution 4.0 (CC-BY) License. Further distribution of this work is permitted, provided the original work is properly cited. 

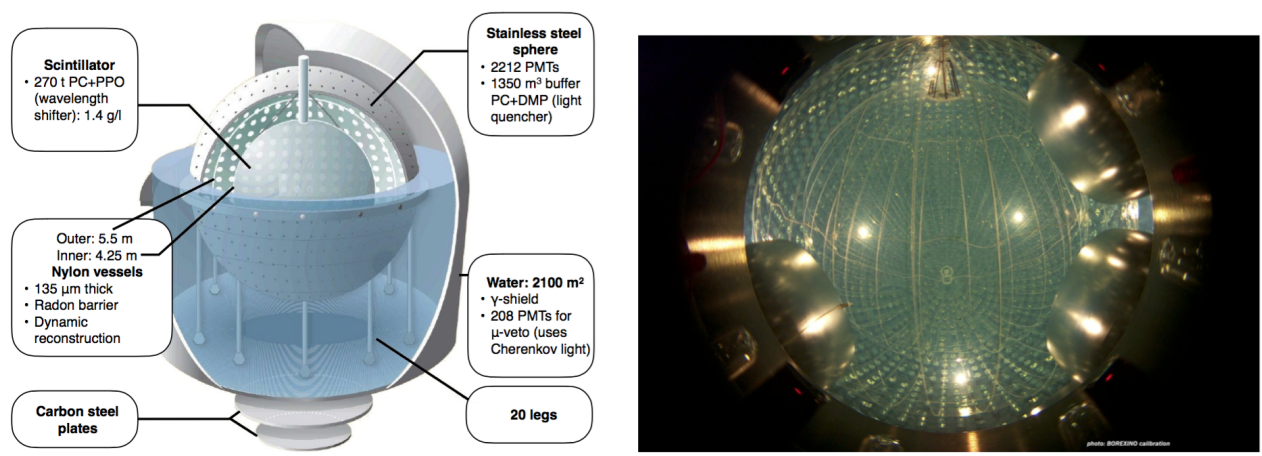

Fig. 1. (Left) Schematics of the Borexino detector. (Right) Picture of the Stainless Steel Sphere interior with visible the Inner and Outer nylon vessels and the PMTs.

spectrum. Borexino has succeeded in determining all major solar neutrino flux components already with its first dataset Phase-I (2007-10): first direct detections of pp ${ }^{2}$, pep ${ }^{3},{ }^{7} \mathrm{Be}^{4}$, the lowest-threshold observation of ${ }^{8} \mathrm{~B}{ }^{5}$ at $3 \mathrm{MeV}$ and the best available limit in the CNO solar flux ${ }^{5}$. More recently, high-precision (down to $2.8 \%$ in the case of ${ }^{7} \mathrm{Be}$ ) determinations of the aforementioned solar neutrino fluxes have been attained using new techniques and enlarged statistics from the post-liquid scintillator purification dataset, Phase-II (December 2011 - May 2016) ${ }^{6}$. Next year, a new calibration campaign in preparation of the Short distance Oscillation with boreXino $\left(\mathrm{SOX}^{7}\right)$ project is scheduled. This project aims to confirm or rule out the presence of sterile neutrinos, which could explain the observed experimental anomalies found at accelerator and reactors ${ }^{8,9,10}$.

Borexino is a neutrino detector based on the principles of ultra-pure liquid scintillator and graded shielding. The overburden of the mountains under which the Laboratori Nazionali del Gran Sasso (LNGS) are located, provide $\approx 3600$ meters of water-equivalent shielding against cosmic backgrounds. The core of the detector is 278 ton of ultra-radiopure organic liquid scintillator (PC+PPO), in a $125 \mu \mathrm{m}-$ thick nylon vessel of $4.25 \mathrm{~m}$ radius, surrounded by 2212 photomultipliers (PMTs). A non-scintillating buffer fills the space between the nylon vessel and a stainless-steel sphere (SSS) of $6.85 \mathrm{~m}$ radius, which supports the PMTs. The entire detector is enclosed in a cylindrical tank filled with ultra-pure water and instrumented with 208 PMTs, acting as an active Cherenkov muon veto and as a passive shield against external $\gamma \mathrm{s}$ and neutrons. The light yield is $\approx 500$ photoelectrons $/ \mathrm{MeV}$, with a hardware threshold of $\approx 50 \mathrm{keV}$. Neutrinos are detected via elastic scattering on electrons in the target material while antineutrinos are detected through the Inverse Beta Decay (IBD) coincidence signal, which is almost background-free.

\section{Solar neutrinos and other results}

The expected solar $\nu$ 's interaction rate in Borexino ranges from few counts to 100 counts per day (cpd) in $100 \mathrm{t}$ depending on the neutrino component. Together with 
the lack of directionality from the scintillation light, this demands a high radiopurity of the detector, a deep understanding of the backgrounds, and an accurate modelling of the detector response. The energy, position, and pulse-shape of each event are reconstructed by exploiting the number of detected photons and their detection times. At $1 \mathrm{MeV}$, the energy and position reconstruction resolution are $\approx 50 \mathrm{keV}$ and $\approx 10 \mathrm{~cm}$ respectively. To analyse data, a selection and software discrimination of events are performed: internal (external) muons are removed, a $300 \mathrm{~ms}$ veto to suppress cosmogenic backgrounds is applied and ${ }^{214} \mathrm{Bi}-{ }^{214} \mathrm{Po}$ fast coincidences from the ${ }^{238} U$ chain and unphysical noise events are removed. In addition, events featuring vertices reconstructed outside a Fiducial Volume (FV) are rejected. FV consists in the innermost region of the scintillator and it is between 70 ton and 100 ton depending on the specific analysis. The residual background is mainly due to radioactive isotopes contaminating the scintillator itself, such as ${ }^{14} \mathrm{C},{ }^{210} \mathrm{Po},{ }^{85} \mathrm{Kr}$ and ${ }^{210} \mathrm{Bi}$ from ${ }^{210} \mathrm{~Pb}$, from the cosmogenic isotope ${ }^{11} \mathrm{C}$ and by residual external background, mainly due to ${ }^{208} \mathrm{Tl}$ and ${ }^{214} \mathrm{Bi}$ (see Fig. 2-left). A novel pulse shape method, based on MultiLayer Perceptron (MLP) machine learning algorithm, has been developed and applied to distinguish between the scintillation pulses of $\alpha$ and $\beta$ particles with high efficiency in the framework of the annual modulation analysis. This analysis algorithm allows to select ${ }^{210}$ Po events with good efficiency. The ${ }^{11} \mathrm{C}$ is generated by muon spallation and has a $\beta$ decay with average time of about 30 minutes, which makes difficult to identify it directly. A statistic reduction of this background can be performed by creating a veto in space and time, associating events to muon-neutron pairs.

An important point is that the Phase-II analysis benefits from the results of an extensive campaign of purification of the scintillator: this included 6 cycles of closedloop water extraction and it has significantly reduced the contribution of some radioactive contaminants: ${ }^{238} \mathrm{U}\left(<9.5 \cdot 10^{-20} \mathrm{~g} / \mathrm{g}\right.$ at $95 \%$ C.L. $),{ }^{232} \mathrm{Th}\left(<7.2 \cdot 10^{-19}\right.$ $\mathrm{g} / \mathrm{g}$ at $95 \%$ C.L.), ${ }^{85} \mathrm{Kr}$ (reduced by a factor $\approx 4.6$ ) and ${ }^{210} \mathrm{Bi}$ (reduced by a factor $\approx 2.3)$.

Four years of Borexino Phase-II data have been analysed to search for the expected annual modulation of the ${ }^{7} \mathrm{Be}$ solar neutrino interaction rate induced by the eccentricity of the Earth's orbit around the Sun ${ }^{12}$. Borexino Phase-II, in addition to higher statistics, lower background levels and an improved rejection of alpha-decay background with respect to Phase-I data, is characterised by the absence of major scintillator handling and thus displays a high degree of stability of the detector, crucially important for identifying time dependent signals.

Three different types of analysis were performed: a sinusoidal fit to the event rate (Fig. 2-right), a spectral analysis with periodic signal assumption (Lomb-Scargle method) and an Empirical Mode Decomposition (similar to Lomb-Scargle but without periodic signal assumption). Amplitude and phase of the modulation are consistent with that expected from the eccentric revolution of the Earth around the Sun, proving the solar origin of the low energy neutrinos detected in Borexino. The absence of an annual modulation is rejected with a C.L. of $99.99 \%$. The direct fit 


\section{Collica}
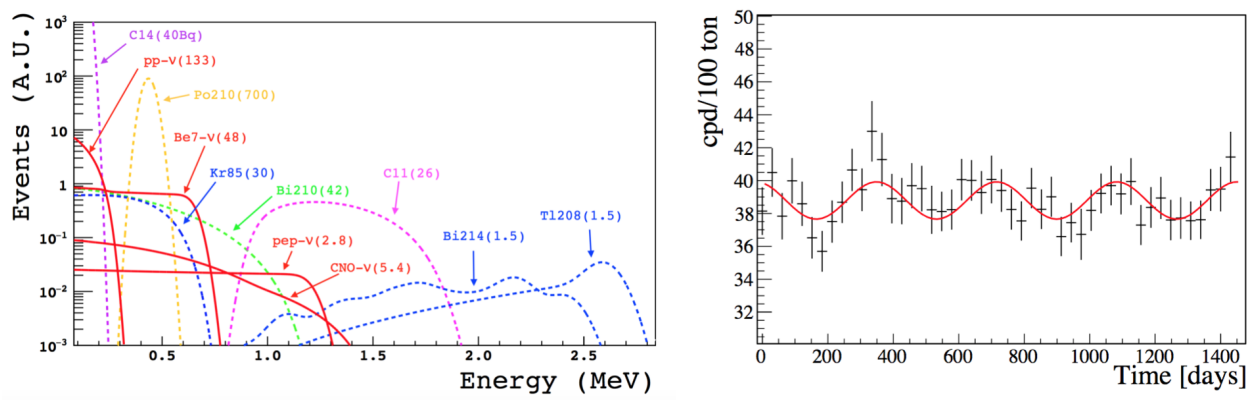

Fig. 2. (Left) Energy spectrum of electron recoils induced by the interaction of solar $\nu$ in Borexino together with the spectral shapes of some known radioactive contaminants in the scintillator. The numbers shown in parenthesis are in cpd per 100 ton for all species apart from ${ }^{14} \mathrm{C}$, for which $\mathrm{Bq}$ per 100 ton is used. (Right) Sinusoidal fit to the measured rate of $\beta$-like events rate.

to the event rate yields an eccentricity of $\epsilon=1.74 \pm 0.45 \%$, while the Lomb-Scargle method identifies a clear spectral maximum at the period $\mathrm{T}=1$ year.

Neutrinos produced in the Sun are a unique source of information with regards to their physical properties. Besides the study of well-established neutrino oscillations they can also be used to look for an anomalous magnetic moment. A search for the solar neutrino effective magnetic moment (NMM) has been performed using the entire Phase-II dataset, namely 1270.6 days. Neutrino-electron elastic scattering is the most sensitive test for NMM search. The NMM implies a small correction in the scattering cross section, therefore a new component in the spectrum appears. The strongest change of shape can be observed in the neutrino flux from the ${ }^{7} \mathrm{Be}$ $\nu$, although the tiny correction on the flux from the pp's $\nu$ can help to constrain the result. Using the recent analysis techniques, an upper limit has been measured: $\mu_{\text {eff }}<2.8 \cdot 10^{-11} \mu_{B}$ (C.L. $\left.90 \%\right)^{13}$, which is slightly better than the current limit of $\mu_{e f f}<2.9 \cdot 10^{-11} \mu_{B}$ set by GEMMA ${ }^{14}$.

The estimated limit can be exploited to put new limits on the magnetic moments of the neutrino flavour states. The effective magnetic moment for the LMA-MSW solution is (assuming that the survival probability of pp and ${ }^{7} \mathrm{Be}$ solar neutrinos is the same):

$$
\mu_{e f f}^{2}=P^{3 \nu} \mu_{e}^{2}+\left(1-P^{3 \nu}\right)\left(\cos ^{2} \theta_{23} \cdot \mu_{\mu}^{2}+\sin ^{2} \theta_{23} \cdot \mu_{\text {tau }}^{2}\right)
$$

where

$$
\begin{array}{r}
P^{3 \nu}=\sin ^{4} \theta_{13}+\cos ^{4} \theta_{13} P^{2 \nu} \\
P^{2 \nu}=\sin ^{4} \theta_{12} \sin ^{2} \frac{\Delta m_{12}^{2} L}{4 E}
\end{array}
$$

$\mathrm{P}$ are the oscillation probabilities, $\theta$ are the mixing angles, $\mathrm{L}$ the distance, $\Delta m_{12}^{2}$ the squared difference masses, E the energy and $\mu$ the magnetic moments for the different flavours. The most conservative case of each flavour contribution can be 

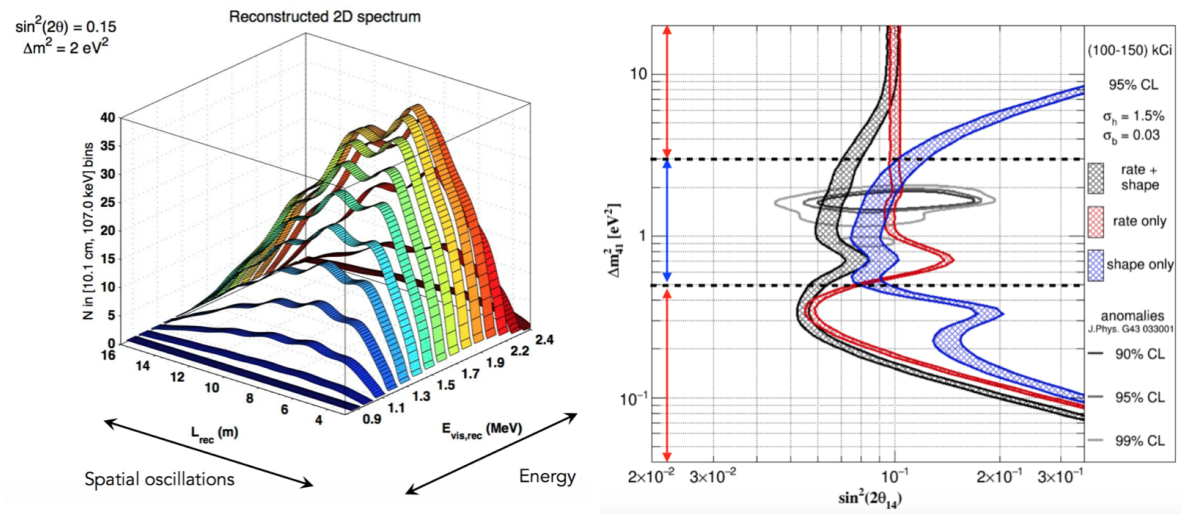

Fig. 3. (Left) Predicted distributions of events as a function of the distance L and the antineutrino energy $\mathrm{E}$, with sterile oscillations and mixing parameters $\sin ^{2}(2 \theta)=0.15$ and $\Delta m^{2}=2$ $\mathrm{eV}^{2}$. (Right) Sensitivity curves: Rate (in red), Shape (in blue) and overall (in black) contributions. Grey contours: preferred region of the anomalous neutrino experiments with C.L.s of 90\%, 95\% and $99 \%$.

obtained by setting other two to zero: the upper limits at $90 \%$ of C.L. are $\mu_{e}<$ $3.9 \cdot 10^{-11} \mu_{B}, \mu_{\mu}<5.8 \cdot 10^{-11} \mu_{B}$ and $\mu_{\tau}<5.8 \cdot 10^{-11} \mu_{B}{ }^{13}$.

\section{Sterile neutrino investigation with SOX}

As hinted in Sect. 1, some experimental anomalies at accelerator and reactors could be solved with the introduction of a light sterile neutrino. Recent results published by Daya Bay experiment ${ }^{15}$ weaken the reactor anomalies, however the accelerator anomalies have still to be understood. The parameter region of interest, $\Delta \mathrm{m}_{14}^{2} \approx 1 \mathrm{eV}^{2}$, can be probed with a short baseline disappearance experiment operating at meter-scale distances and MeV-scale energies. SOX ${ }^{7}$ has been designed to investigate the existence of a sterile neutrino by measuring the antineutrino flux coming from $\mathrm{a} \approx 100 \mathrm{kCi}{ }^{144} \mathrm{Ce}-{ }^{144} \mathrm{Pr}$ antineutrino generator (CeANG) positioned in a pit under the Borexino detector, at a distance of about $8.5 \mathrm{~m}$ from its center. The antineutrinos produced by CeANG have a continuous spectrum with an endpoint at $2.99 \mathrm{MeV}$. This allows to investigate a larger parameter space if the energy is reconstructed with precision. The lifetime of ${ }^{144} \mathrm{Ce}$ is about 400 days and about 10000 events are expected in 1 year and an half of data taking.

Anti-neutrinos are detected inside Borexino via inverse beta decay, providing a clear coincidence signature with a prompt positron annihilation and a delayed neutron capture ${ }^{11}$. Two methods will be used for the analysis: the standard disappearance technique and the oscillometry measurement within the detector volume (see Fig. 3-left). For the first technique, the CeANG activity must be known with precision and a calorimetric measurement will be employed ${ }^{16}$. For the second technique, the precise knowledge of the activity is not necessary since a possible oscillation pattern would be visible independently of any activity estimate. 


\section{Collica}

The sensitivity of SOX is expected to be maximised when the two techniques are combined together: by assuming $\sigma=1.5 \%$ on the activity measurement and $\sigma=0.03$ on the spectrum measurement, almost the whole anomaly region could be probed (see Fig. 3-right).

To reach a deep understanding of the energy response and spatial reconstruction in the whole active volume, a calibration campaign of the Borexino detector is needed ${ }^{17}$. It will be performed with a suite of low-activity radioactive sources and the calibration system, already used in Borexino Phase-I, allows to insert the sources without perturbing the radio-purity of the detector. The calibration campaign will take place a few months before the beginning of the SOX project, scheduled to start in April 2018.

\section{Acknowledgements}

The Borexino program is made possible by funding from INFN (Italy); the NSF (U.S.); BMBF, DFG, (HGF, and MPI (Germany); RFBR (Grants No. 15-0202117, No. 16-29-13014, No. 16-02-01026 and No. 17-02-00305), RSF (Grant No. 1712-01009) (Russia); NCN Poland (Grant No. UMO-2013/10/E/ST2/00180); FNP Poland (Grant No. TEAM/2016-2/17). We acknowledge the generous support and hospitality of the Laboratori Nazionali del Gran Sasso (LNGS).

\section{References}

1. G Alimonti et al. (Borexino Coll.), Nucl. Instr. Meth. A 600, arXiv:0806.2400, 2011

2. G Bellini et al. (Borexino Coll.), Nature 512, 383-386, 2014

3. G Bellini et al. (Borexino Coll.), Phys. Rev. Lett. 108, arXiv:1110.3230, 2012

4. G Bellini et al. (Borexino Coll.), Phys. Rev. Lett. 107, arXiv:1104.1816, 2011

5. G Bellini et al. (Borexino Coll.), Phys. Rev. D 82, arXiv:0808.2868, 2010

6. M Agostini et al. (Borexino Coll.), arXiv:1707.09279v1, 2017

7. G Bellini et al. (Borexino/SOX Coll.), JHEP 38 1308, arXiv:1304.7721, 2013

8. A Aguilar et al. (LSND Coll.), Phys. Rev. D 64 112007, arXiv:hep-ex/0104049, 2001

9. A Aguilar et al. (MiniBooNE Coll.), Phys. Rev. Lett. 110 161801, arXiv:1303.2588, 2013

10. G Mention et al., Phys. Rev. D 83 073006, arXiv:1101.2755, 2011

11. M Agostini et al. (Borexino Coll.), Phys. Rev. D 92 031101, arXiv:1506.04610, 2015

12. M Agostini et al. (Borexino Coll.), Astropart. Phys. 92, 21 arXiv:1701.07970, 2017

13. M Agostini et al. (Borexino coll.), Phys. Rev. D 96, 091103 arXiv:1707.09355, 2017

14. A.G. Beda, V.B. Brudanin, V.G. Egorov, D.V. Medvedev, V.S. Pogosov, E.A. Shevchik, M.V. Shirchenko, A.S. Starostin, I.V. Zhitnikov, Phys. Part. Nucl. Lett. 10, 139 (2013)

15. F P An et al. (Daya Bay Coll.), Phys. Rev. Lett. 118 251801, arXiv:1704.01082, 2017

16. L Papp et al., Nucl. Instr. Meth. A 824, 2016

17. L Collica, D Bravo Berguno, K Choi and M Nielsony (for the Borexino/SOX Coll.), Proceedings of the 2017 Topics in Astroparticle and Underground Physics, IOP Press, publication in 2018 